zwischen den Grenzwerten adiabatisch $(\boldsymbol{y}=\infty)$ und isotherm $(v=0)$ restiert, wenn die äwBere Behinderung von Schwingungen durch Arbeiten im Vakuum eliminiert ist. - Das Gebiet mittlerer Relaxationszeiten kann man statistisch durch Spannungssprünge darstellen, deren Eintreffen von der Vorgeschichte, d. h., den schon früher erfolgten Spannungsänderungen $a b$ hängt ( $J \cdot K u b a ́ t)$. Man erhält eine Zeitfunktion, welche als Grenzwert für kleine Relaxationszeiten die Maxwellsche Beziehung und als Grenze für große Relaxationszeiten die bekannte lineare Funktion zwișchen Spannung und dem Logarithmus der Zeit ergibt. - Von Wo. Ostwalds Einfluß der „mecha. nischen Vorgeschichte" führt ein Weg bis zu den „rheologischen Verteilungsfunktionen" (B. Gross), in welchen eine Kombination der Kriechkurve mit der Erschlaffungskurve erfolgt, wobei durch Überlagerung der Funktionen verschiedene Deformations-Zeit-Beziehungen abgeleitet werden, von ideal elastischen und rein viskosen Systemen angefangen, bis zu solchen mit „Gedächtnis“.

Außer diesen Vorträgen zur allgemeinen Rheologie wurden auch speziellere Themen behandelt. Die thixotropen Erscheinungen lassen sich in das Gesamtschema rheologischer Moglichkeiten eingliedern, wenn man den Dispersitätsgrad der dispersen Phase neben der Fließeigenschaft des Dispersionsmittels als Parameter einführt (J. Pry ce-Jones). - Umgekehrt eröffnet die Diagnose der Strukturviskosität makromolekularer Lösungen (Zellulose in Butylacetat) weitere Möglichkeiten zu einer absoluten viskosimetrischen Molekulargewichtsbestimmung solcher Systeme (K. Edelmann).Mechanische Schwingungen $(100 \mathrm{~Hz})$ verringern die innere Reibung thixotroper Systeme. Sie setzen auch die äußere Reibung disperser Systeme auf ihrer Un terlage herab und geben damit Anlaß zu entsprechen. den Fließbewegungen und Klassierungsvorgängen ( $R$. A uerbach).

Die Vorträge auf dem Gebiete der angewandten Rheologie bezogen sich auf das Anstrichgebiet (G., Zeidler), auf Schmiermittelreibung (S. v. Schiessl) wobei mittels einer optischen Interferenzanalyse des Abreißvorganges zweier Metallplatten auf eine haftende Ölschicht in der Größenordnung von $\mu$ geschlossen wurde. Sie leiten über zu den Vorträgen rein methodischen Inhaltes, die sich auf Meßmethoden an flüssigen und konsistenten Stoffen ( $\mathrm{Sch}$ waben/Umstät ter, Höppler, Göttner/Schultze), auf röntgenographische Bestimmung von Verformungsvorgängen an Metallen (O. V a u peI) und auf Dehnungsmessungen an festen Stoffen durch elektrische Widerstandsänderung eines aufgebrachten MeßBstreifens (H.H. Ems c her $\operatorname{man} n$ ) beziehen.

Die Tagung unter der Leitung von Ubbelohde und Umstätter wurde durch einen Begrüßungsabend am 24. 9. 1952 im Parkhotel in Dahlem eingeleitet und endete mit einer Mitgliederversammlung am 27. 9. 52 im Materialprüfungsamt, wo auch die Vorträge stattfanden. Insgesamt hat sie gezeigt, daß die Rheologie, ähnlich wie die Kolloidchemie, zu einer neuen und produktiven $Q u e r v e r b i n d u n g$ verschiedenster Disziplinen führt, die von der theoretischen Physik bis zur Nylonfaser und von der Physiologie bis zur Geologie reicht. Ihre Vertreter sprechen daher vorläufig noch eine recht verschiedene Sprache.

\title{
Berichtigung
}

\section{Über den Mechanismus der aggregierenden Wirkung von Mehl oder Stärke auf das disperse System Rotschlamm in Aluminatlösung}

\author{
Von I. Dvornik und E. Herrmann (Zagreb) \\ Kolloid-Z. 128, 75 (1952)
}

Auf Seite 76 wurden die Pfeile in der Tabelle 1 waagrecht statt senkrecht eingesetzt, richtig muß es sein :

\section{Tabelle I}

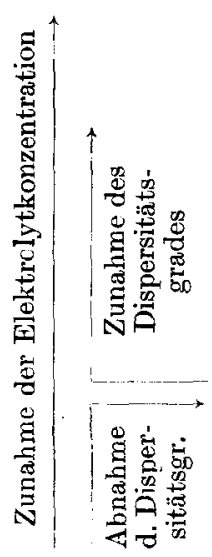

Das Verhalten des Rotschlamms in Abhängigkeit von der Konzentration der Aluminatlösung

$175-250 \mathrm{~g} \mathrm{Al}_{2} \mathrm{O}_{3} / 1$ (und mehr) $\rightarrow$ Stark solvatisiertes System. Rotschlamm und Dispersionsmittel besitzen eine zusammenhängende ,Struktur“. Geringe Neigung zur Aggregierung.

$150-175 \mathrm{~g} \mathrm{Al} l_{2} \mathrm{O}_{3} / \mathrm{l} \rightarrow$ Beginnende Aggregierungstendenz. Als Vorstufe der Aggregierung tritt "Schwarmbildung“ auf. Ansteigen der scheinbaren relativen Viskosität $\left(\eta_{r} \mathrm{e}_{l}\right)(7)$.

$10-150 \mathrm{~g} \mathrm{Al}_{2} \mathrm{O}_{3} / \mathrm{l} \rightarrow$ Mit fallender Konzentration dauernde Zunahme der Aggregierbarkeit. Der Schlamm besitzt die Eigenschaften eines Suspensoidgels.

$5-10 \mathrm{~g} \mathrm{Al}_{2} \mathrm{O}_{3} / \mathrm{l} \rightarrow$ Maximum der Größe der Aggregate, Minimum des Sedimentvolumens, Minimum der Solvatation.

1,6-5,0 $\mathrm{g} \mathrm{Al}_{2} \mathrm{O}_{3} / \mathrm{l} \rightarrow$ Beginnende Dispergierung der Aggregate.

$1,5-2,2 \mathrm{~g} \mathrm{Al}_{2} \mathrm{O}_{3} / 1 \rightarrow$ Anfangseffekt der Peptisation.

$0,6-1,4 \mathrm{~g} \mathrm{Al}_{2} \mathrm{O}_{3} / 1 \rightarrow$ Haupteffekt der Peptisation. 